\title{
INVESTIGATIONS ON NONLINEAR DEFORMATION PHENOMENA IN A FORCE CALIBRATION SYSTEM
}

\author{
Y. Wang ${ }^{1}$, N. Rogge ${ }^{2}$, S. Vasilyan ${ }^{2}$, T. Fröhlich ${ }^{2}$ \\ ${ }^{1}$ Tianjin University, Tianjin 300072, China, 3016202030@tju.edu.cn \\ ${ }^{2}$ Technische Universität Ilmenau, 98693 Ilmenau, Germany, norbert.rogge@ @u-ilmenau.de
}

\begin{abstract}
:
Previous investigations show, in force calibration and measurement systems that are based on electromagnetic force compensation (EMFC), deformations of the flexure hinges can cause significant contributions to the uncertainty of the system. A Simulink model of the Planck-Balance 2 (PB2) is established in MATLAB according to the $\mathrm{CAD}$ model. The results are compared to simplified analytical models and the results obtained from measurement.
\end{abstract}

Keywords: Planck-Balance; EMFC balance; mechanical nonlinearities; mechanical simulation; force calibration

\section{INTRODUCTION}

EMFC balances are state of the art for weighing systems with low uncertainties. However, they can also be modified and used as force measurement systems. A recent example is the calibration of laser power by the measurement of the photon momentum [1]. Another application, where a modified EMFC load cell is used, is the PlanckBalance (PB). It is a table top Kibble balance that was developed in cooperation between the Physikalisch-Technische Bundesanstalt (PTB) and the Technische Universität Ilmenau. In contrast to a standard EMFC balance, an interferometer and a second voice coil actuator are attached to the load carrier. The additional actuator is calibrated in the velocity mode and utilized in the force mode to compensate the gravitational force of the test weight [2].

The problem that is addressed in this work will be exemplary shown for the PB2 system. This system is the version of the Planck-Balance that aims to achieve uncertainties suitable for calibration class $E_{2}$ mass standards in the range from $1 \mathrm{mg}$ to $100 \mathrm{~g}$. In the force mode of the current PB2 system, the gravitational force is compensated by an external voice coil actuator that is attached to the load carrier of the system, while the position that indicates a force equilibrium is generated by the internal position sensor of the balance. Preliminary investigations showed that the there is a different displacement on the load carrier side of the lever depending on the load that is placed onto it. As expected, these displacements are smaller than in the normal use of the EMFC balance where the compensation force is generated at the position sensor side of the lever but, as shown in Figure 1, they can be nonlinear towards the applied load.

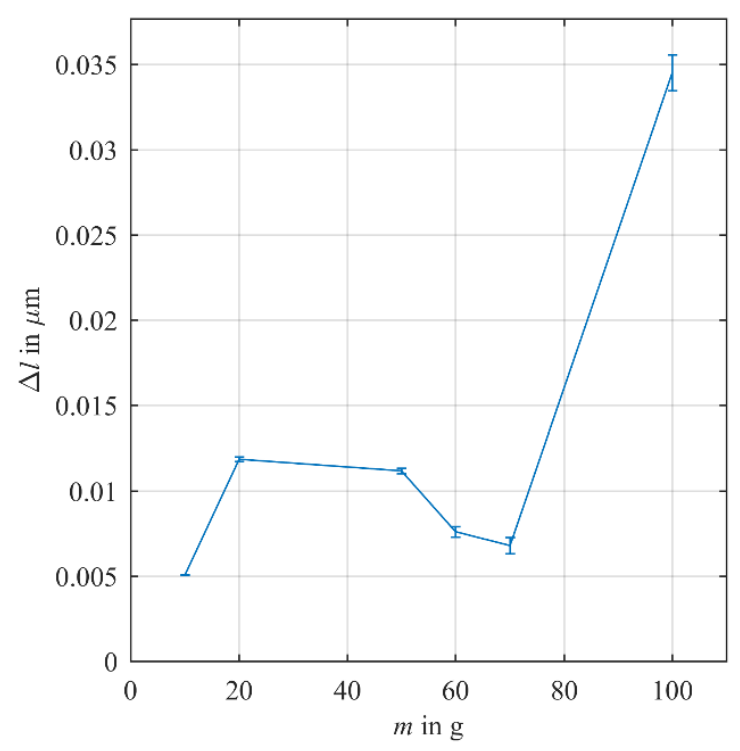

Figure 1: Difference of load carrier displacement for load changes of different masses

The shown difference of the displacement between loaded and unloaded state is measured by the interferometer of the PB2 system, where the error bars represent the standard deviation of the repeated determinations of this difference.

The models that are presented in this work are used to identify how deformations of the mechanical system contribute to the observed nonlinear behaviour. Furthermore, an improved understanding of the effects will help to improve the design of the system and the correction of errors caused by the deformation of the mechanical subsystem. 


\section{MODEL OF THE BALANCE}

In order to investigate the nonlinear relation between mass and load carrier displacement, a rigid body model based on the one described in [3] is set up and modified. The model focuses on the investigation of geometrical nonlinearities, which are able to cause the observed deformation effects. The mechanical model is extended by the electronic hardware and the PID algorithm, which is used to balance the transmission lever after a change of the external mass on the weighing pan (see Figure 2). The model is mainly made up of five sections: mechanical system, position sensor, PID controller, $\mathrm{U} / \mathrm{I}$ converter and voice coil actuator.

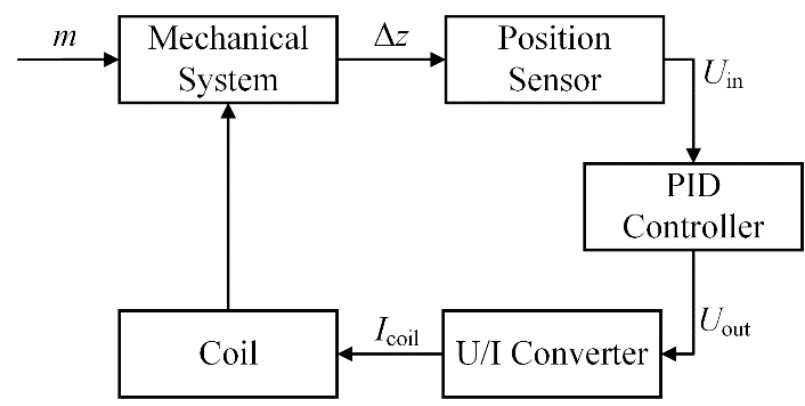

Figure 2: Main components of the balance model

\subsection{Mechanical Subsystem}

The mechanical system is based on the CAD model and mechanical drawings of the device.

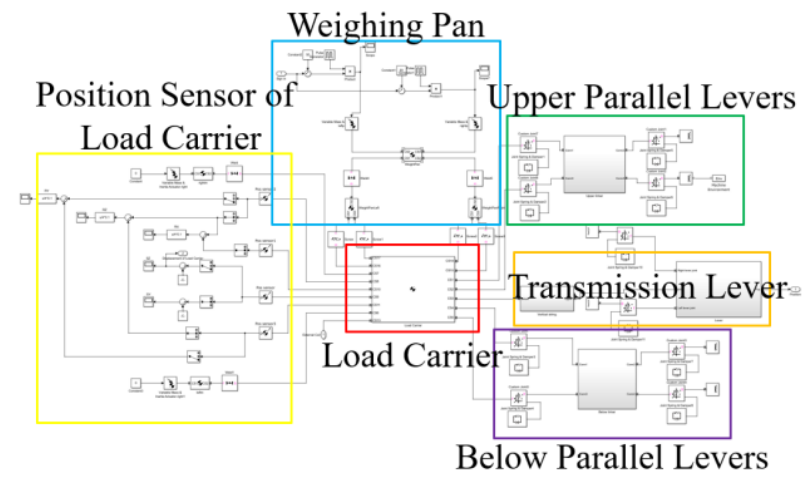

Figure 3: Simulink model of the mechanical subsystem

The design of the mechanical system module is shown in Figure 3. The simulation environment is ideal. The value of the acceleration of gravity $g$ is taken from a previous measurement, and the effects of temperature, humidity or air damping in the environment are not considered.

The damping of Planck-Balance during the measurement mainly comes from the following three sources: the friction of the flexure hinge, air friction and electromagnetic damping of the coil. Compared to the other two types of damping, the air friction damping has a relatively small effect and can be ignored. The electromagnetic damping will be discussed later. The damping caused by the friction force in the flexure hinge is corrected by an empirical value obtained from fitting the experimental data to the simulation result.

The position sensor is placed at the end of the transmission lever in the Planck-Balance mechanical system, in order to detect the displacement of the transmission lever. Its characteristics are obtained from experimental data and a saturation is introduced to the model to represent the mechanical end stops.

\subsection{PID Controller}

The parameter setting of this module mainly refers to the Ziegler-Nichols method [4]. At first, set both the integral parameter $K_{\mathrm{I}}$ and the differential parameter $K_{\mathrm{D}}$ to zero. Then adjust the proportional parameter $K_{\mathrm{P}}$ from small to large until the stable oscillation occurs. Read the $K_{\mathrm{P}}$ value at this time and record it as $K_{\mathrm{u}}$. Record the oscillation period as $T_{\mathrm{u}}$. Then calculate the theoretical parameter values of $K_{\mathrm{P}}, K_{\mathrm{I}}$ and $K_{\mathrm{D}}$ according to the Ziegler-Nichols method parameter table. Finally, adjust the parameters based on the simulation results.

\subsection{Voice Coil Actuator}

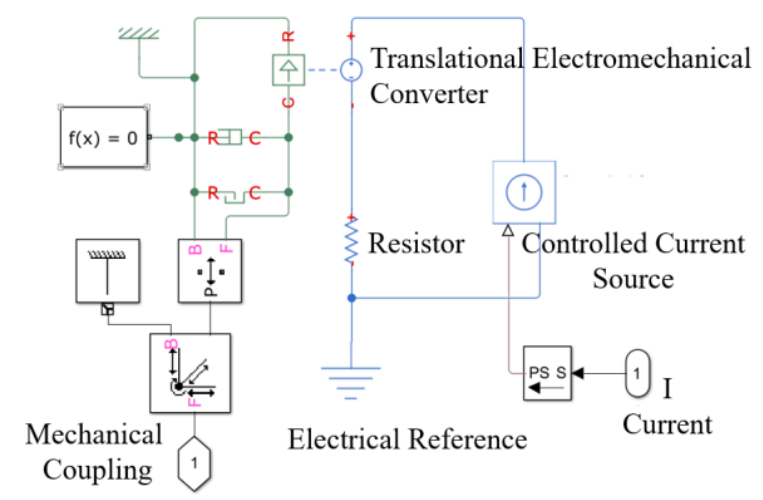

Figure 4: Simulink model for voice coil actuator

The Simulink model of the voice coil actuator is shown in Figure 4. In terms of the working principle, the module is a voice coil actuator, which can convert the current signal input into an electromagnetic force. The output is returned to the load carrier in the mechanical system module to achieve a closed loop of the entire system. The module consists of two parts: coil and permanent magnet. The coil moves in the magnetic field generated by the permanent magnet, and the force generated by the coil actuator compensates for the gravitational force of the load carrier and the weight to be measured, which makes the PB2 system balance.

The force on the electromagnetic coil is the Lorentz force, which is the force generated by the interaction of the current flowing through the electromagnetic coil in the magnetic field. At the same time, when the coil moves in the magnetic field, a dynamic electromotive force is also generated inside the coil. 
$F=I \cdot l \times B$.

$V=u \cdot l \times B$.

$F$ - Lorentz force

$I$ - Current flowing through the solenoid

$l-$ Voice coil winding length

$B$ - Magnetic induction

$V$ - Dynamic electromotive force

$u$ - Velocity of coil

The parameter $K$ needs to be set. $K$ is the cross product of the coil winding length $l$ and the magnetic field magnetic induction $B$, and the unit is N/A or $\mathrm{V} /(\mathrm{m} / \mathrm{s})$. In this model, the value of $K$ is 189 N/A. As for the internal actuator, the value of $K$ changes to -50 N/A. Another parameter that plays an important role during the motion of the coil is the translational damping coefficient. It is determined as $9 \mathrm{~N} /(\mathrm{m} / \mathrm{s})$ by fitting the model behaviour to the experimental data.

The Simulink model of the voltage/current converter (U/I Converter) is equivalent to a $500 \Omega$ resistor. When the input voltage signal $U$ is within the set saturation voltage range, the output current signal can be equivalent to the current signal passing through the $500 \Omega$ resistor. The purpose of setting the saturation value of the voltage signal is to prevent the output current signal from being too large, which will cause a large deflection of the Planck-Balance module. This setting improves the stability of the simulation model.

\section{RESULT OF SIMULATION}

Based on the analysis of the working principle of Planck-Balance, the Simulink model of PlanckBalance is established in MATLAB according to the CAD model, including the mechanical structure subsystem, position sensor, U/I converter, electromagnetic coil actuator and PID controller. The simulation model can be used to study the movement of the model in several non-ideal situations.

\subsection{Ideal Condition}

The simulation model can accurately represent measurement process of the Planck-Balance. Under ideal simulation conditions, a measurement cycle of $20 \mathrm{~s}$ is used. In the first half of the cycle, the weighing pan is unloaded. In the second half of the cycle, the weighting pan is loaded. The movement of the load carrier is observed and the output voltage of the PID controller is recorded.

The displacement of the position sensor oscillates when the load alternates to unload and then quickly returns to zero. The output voltage of the PID controller is stable at unload and load respectively. The voltage difference between the two is proportional to the mass of the weight. The load carrier displacement for loaded and unloaded state of the balance is shown in Figure 5. Similar to the observed behaviour of the real PB2 system, both states show a different displacement, but the difference is smaller than the measured one.

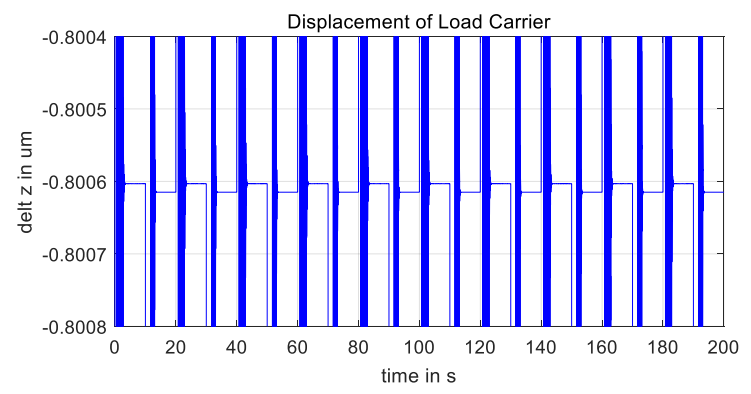

Figure 5: Displacement of load carrier

\subsection{Bending of the Transmission Lever}

In the actual measurement process, the transmission lever will bend due to the influence of gravity. In order to study the influence of this deformation on the measurement, in this section, the transmission lever will be bent by applying an external force. Then, the movement of the load carrier is observed and recorded.

\subsubsection{Applying external force on one side of the balance}

The simple model shown in Figure 6 describes the working state of the balance. $G$ represents the gravity of the weight to be tested placed on the load tray, and $F$ represents the force exerted on the load carrier. The default direction is the same as the direction of gravity. The right side of the balance model represents the position sensor. When the left side of the balance is forced to move, the right side of the balance will produce a corresponding reverse movement. At the same time, the position sensor converts the detected position offset signal into a voltage signal and inputs it to the PID controller. At the same time, the output voltage of the PID controller generates via the voltage-current converter (not shown in the simple model) an output current signal that enters the external voice coil actuator. So, the coil generates an upward electromagnetic force to balance the weight and the additional applied force. Finally, the position sensor is kept in the zero plane and the balance is balanced.

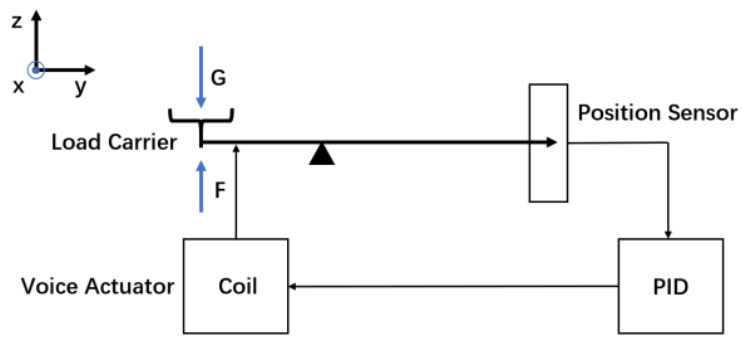

Figure 6: A simple model of the PB2 (Model A)

A different model is shown in Figure 7, an internal coil actuator is used. The torque generated 
by the actuator acts on the end of the transmission lever.

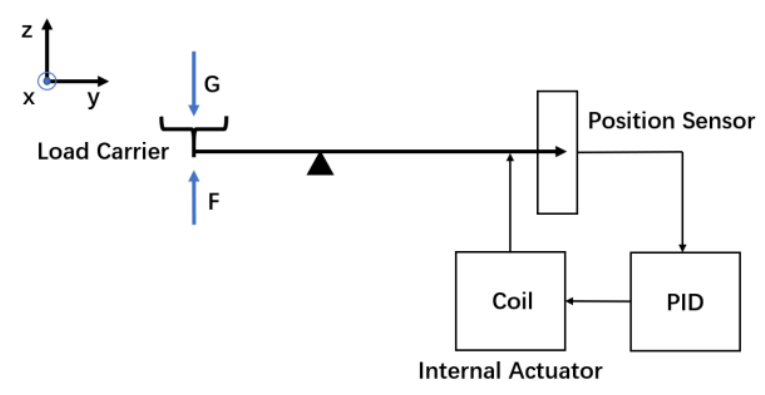

Figure 7: Model with internal actuator (Model B)

Figure 8 shows a simpler Planck-Balance model. The end of the transmission lever is fixed by a mechanical method to keep it in zero plane all the time.

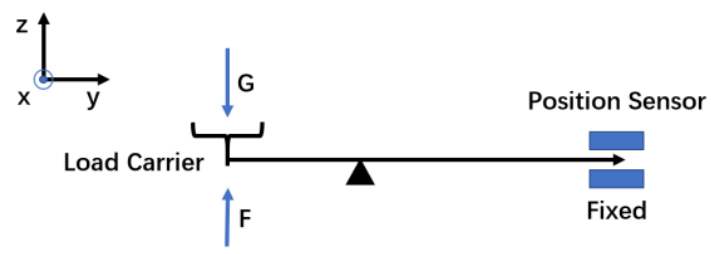

Figure 8: Fixed transmission lever (Model C)

In the simulation, the external force $F$ applied by the facility to the load carrier is $5 \mathrm{~N}$, and the direction is opposite to the direction of the weight $G$. The three models were used for six sets of experiments. The weights used in the six sets of simulation were $10 \mathrm{~g}, 20 \mathrm{~g}, 50 \mathrm{~g}, 60 \mathrm{~g}, 70 \mathrm{~g}$ and $100 \mathrm{~g}$. Each set of simulation measures ten cycles of data, each cycle lasting $20 \mathrm{~s}$. In the first half of the cycle, the weighing pan is unloaded. In the second half of the cycle, the weighing pan is loaded and is subjected to an external vertical upward force.

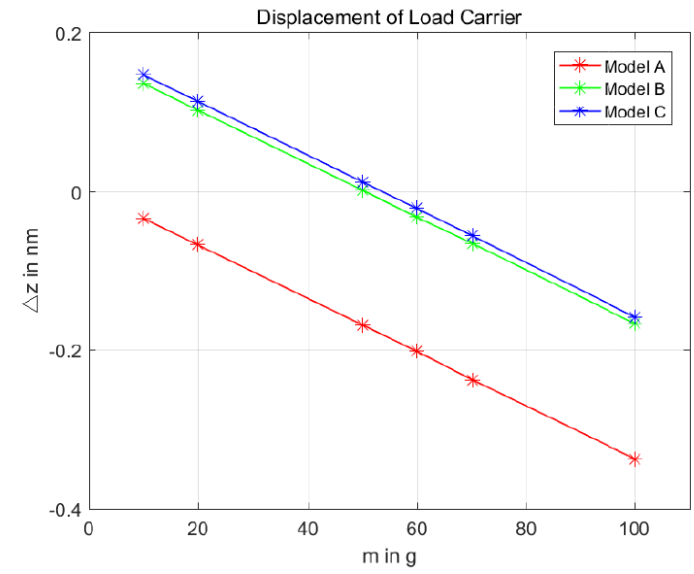

Figure 9: Difference of displacement in Model A/B/C

As shown in Figure 9, the red line represents the simulation result of Model A. It is not difficult to find that the extension line of the red line segment crosses the $(0,0)$ point, which means that under this condition, the displacement of the load carrier is proportional to the mass of the weight. Model B shows only the difference that all data has a constant offset from the data of the classic model A. The blue line represents the simulation result of model $\mathrm{C}$, which is almost the same as the result of model B. Within the acceptable range of measurement error, it can be considered that fixing the end of the transmission lever by mechanical method and using the internal actuator has the same effect on the mechanical deformation of the measuring system. This is one of the reasons why the external electromagnetic coil driver is selected in the actual measurement process. The use of the external actuator can make less deformation of the mechanical structure during the measurement process and can be closer to the ideal state. It can reduce measurement errors, improve measurement capabilities and resolution.

\subsubsection{Apply external force on both sides of the balance}

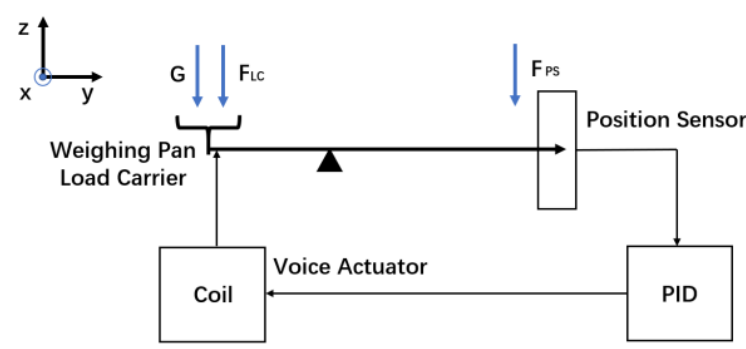

Figure 10: Schematic of the external force applied at both ends of the balance

In order to make the transmission lever have a bigger deformation, two forces in the same direction to both ends of the transmission lever are applied. One force is applied to the centre of gravity of the load carrier, and the other force is applied to the end of the transmission lever. The force applied by the facility to the load carrier is $F_{\mathrm{LC}}$, and the force applied to the end of the transmission lever is $F_{\mathrm{PS}}$. The direction of these two forces is the same as the direction of gravity, and

$F_{\mathrm{LC}}=3.75 \cdot F_{\mathrm{PS}}$.

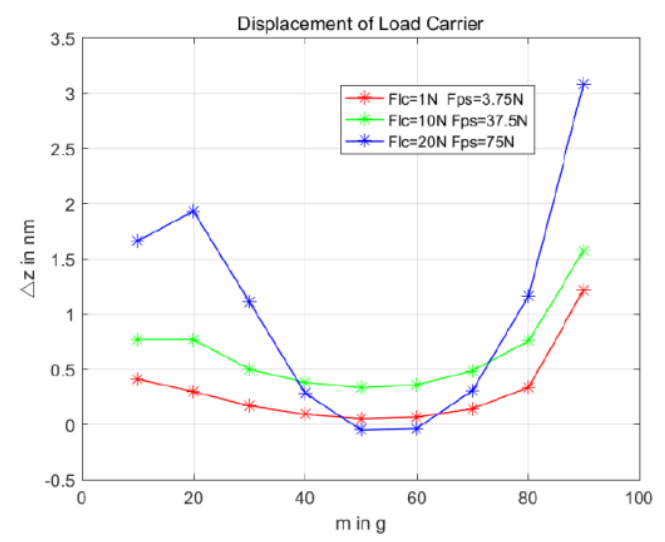

Figure 11: Difference of displacement of load carrier when external force is applied at both ends 
The simulation results are shown in Figure 11. The abscissa indicates the mass of the measurement weight in grams. The ordinate is the displacement of the load carrier in nanometres. The red line represents the result of the Simulink model when $F_{\mathrm{LC}}=3.75 \mathrm{~N}$ and $F_{\mathrm{PS}}=1 \mathrm{~N} ;$ the green one represents $F_{\mathrm{LC}}=37.5 \mathrm{~N}$ and $F_{\mathrm{PS}}=10 \mathrm{~N}$; the blue one represents $F_{\mathrm{LC}}=75 \mathrm{~N}$ and $F_{\mathrm{PS}}=20 \mathrm{~N}$. The measurement results show that when the transmission lever bends due to the force on both sides, the movement of the load carrier will show nonlinearity, and the greater the degree of bending, the more prominent the nonlinear behaviour. However, even when the force is increased to values of $F_{L C}=75 \mathrm{~N}$ and $F_{P S}=20 \mathrm{~N}$, the maximum external displacement of the load carrier still retains at about $3 \mathrm{~nm}$, smaller than observed in measurement. Therefore, it is considered that the bending of the transmission lever is not the main reason for the non-linear deformation in the force calibration system.

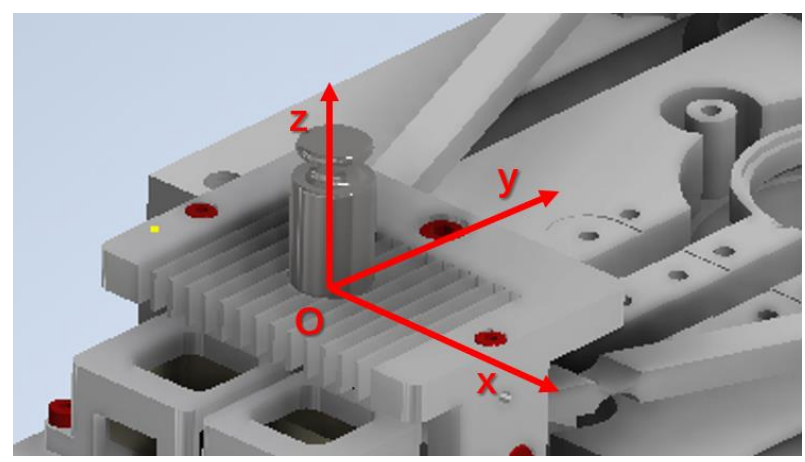

Figure 12: Coordinate system at the centre of the weighing pan

\subsection{Off-Centre Load}

In the actual measurement process, the weight is picked up by the experimenter with tweezers or a mechanical arm and placed on weighing pan. In this process, there is no guarantee that the weights are placed exactly in the centre of the pan, which may cause the nonlinear displacement of the load carrier during the measurement. In order to study the influence of this phenomenon, as shown in Figure 12, a plane coordinate system is established on the surface of the weighing pan, and the $\mathrm{x}$-axis and $\mathrm{y}$ axis are set according to the illustrated direction. In the simulation results that are shown in this section, the independent variable is the position of the weight. The object of observation is the displacement difference between load and unload. As in the experiment, this displacement is obtained at a position that is offset from centre of gravity of the load carrier since the beam of the interferometer cannot be pointed there due to design restrictions. Therefore, the tilt of the load carrier due to a deformation of the mechanical system has an important role in the described phenomena.

\subsubsection{Y-axis Offset}

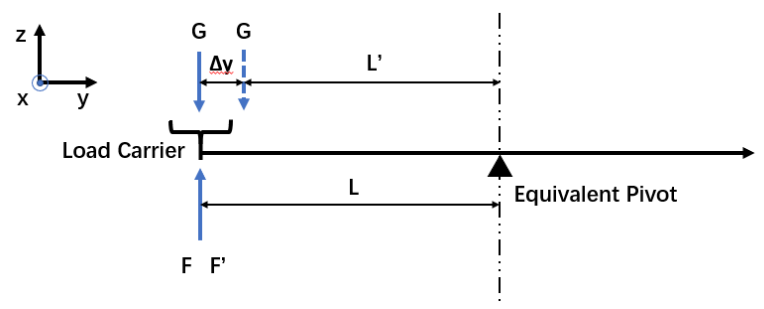

Figure 13: The weight is offset in $y$-axis direction

As shown in Figure 13, when the weight is placed in the centre of the weighing pan, the gravity $G$ is equal to the force $F$ generated by the external actuator.

$F=G$.

When the position of the weight is shifted in the y-axis direction, the gravity of weight is still $G$. The difference is that the equivalent force arm is $L^{\prime}$, where $L^{\prime}=L-\Delta y$, and the support force provided by the actuator at this time is $F$, according to the principle of lever balance.

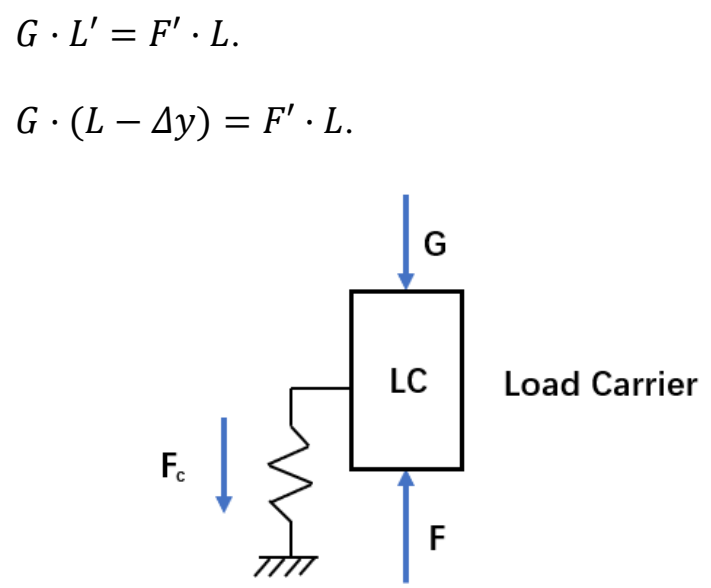

Figure 14: Simple spring model of load carrier

In Section 3.2, we discussed that when the right side of the balance is fixed, the left side can still balance the weight of the weight through its own deformation without external force. Therefore, the right side of the balance can be considered as a simple spring model. As shown in Figure 14, set the spring coefficient of the model to $C$. Then the support force $F_{\mathrm{c}}=C \cdot \Delta Z$ is generated when the load carrier is displaced in the z-axis direction. For the force balance of the load carrier on the $\mathrm{z}$-axis.

$G+F_{C}=F^{\prime}$.

So

$\Delta Z=-(G \cdot \Delta y) /(L \cdot C)$.

It can be obtained from equation (8) that in the above model, the additional displacement of the load carrier will be in accordance with the weight $G$ of the weight and the deviation $\Delta y$, where $L$ and $C$ are constants. The gravitational force $G$ and the 
deviation $\Delta y$ are used as variables to observe the movement of the load carrier during the measurement process.

Figure 15 shows the displacement of load carrier in different deviation $\Delta y$. It was generated with the Simulink model which does not show measured data. The results verify the rationality of the simple spring model as well as the correctness of equation (8) in an intuitive and simple way. The slope $\mathrm{G} /(\mathrm{L} \times \mathrm{C})$ can be identified from Figure 15, then the value of $\mathrm{C}$ can be determined.

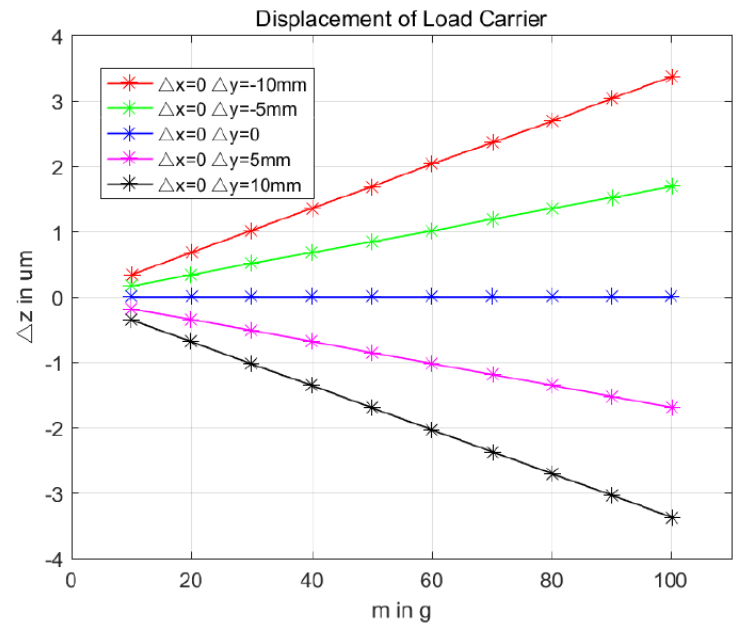

Figure 15: Displacement of the load carrier when the weight is offset in y-axis direction

\subsection{2. $\mathrm{X}$-axis Offset}

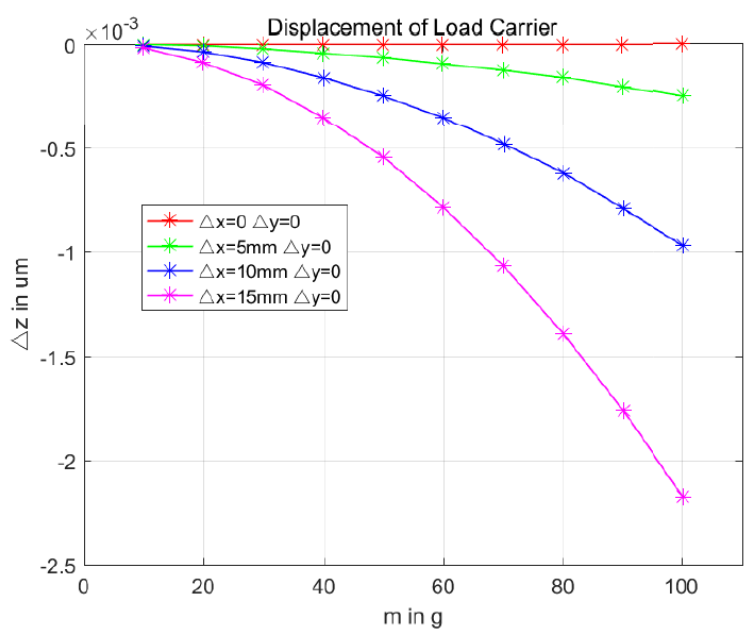

Figure 16: Displacement of the load carrier when the weight is offset in $\mathrm{x}$-axis direction
As shown in Figure 16, the offset of the weight position on the $\mathrm{x}$-axis will also cause additional displacement of the load carrier, but it is much smaller than the situation when y-axis is offset. Therefore, it is considered that when the weights are offset on the $\mathrm{x}$-axis and the $\mathrm{y}$-axis at the same time, the $y$-axis offset is the main reason for the additional displacement of the load carrier.

\section{SUMMARY}

The investigations show that nonlinear deformations can occur in EMFC balances. A rigid body model based on previous work about modelling of the dynamic characteristics of the same balance is proposed in order to investigate the contribution of geometrical nonlinearities to the observed effects.

The simulation results show that the weighing pan will have additional displacement under several conditions such as bending of the transmission lever, eccentric position of the weight, which influence the relation between mass and load carrier displacement that shows nonlinear pattern. However, these contributions above are much smaller than the deviation in the experiment. These factors are responsible for the nonlinear deformation in a force calibration system, but not the main reason. Therefore, the basic factor which causes a dominant performance in the deviation phenomenon still needs further investigation.

\section{REFERENCES}

[1] E. Manske, T. Fröhlich, S. Vasilyan, "Photon momentum induced precision small forces: a static and dynamic check", Measurement Science and Technology, vol. 30, 105004, 2019.

[2] C. Rothleitner et al., "The Planck-Balance - using a fixed value of the Planck constant to calibrate $\mathrm{E}_{1} / \mathrm{E}_{2}$-weights", Measurement Science and Technology, vol. 29, no. 7, 2018.

[3] H. Bai, N. Rogge, C. Rothleitner, T. Fröhlich, "Model based correction of motion deviations in the Planck-Balance", GMA/ITG-Fachtagung Sensoren und Messsysteme, vol. 20, pp. 554-560, 2019.

[4] J. G. Ziegler, N. B. Nichols "Optimum setting for automatic controllers”, Trans. ASME, vol. 64, pp. 759-768, 1942. 